\title{
Symptoms of depression in a large healthy population cohort are related to subjective memory complaints and memory performance in negative contexts
}

\author{
S. Schweizer ${ }^{1 *}$, R. A. Kievit ${ }^{1}$, T. Emery ${ }^{1}$, Cam-CAN $^{2}$ and R. N. Henson ${ }^{1}$ \\ ${ }^{1}$ Medical Research Council Cognition and Brain Sciences Unit, Cambridge, UK \\ ${ }^{2}$ Cambridge Centre for Ageing and Neuroscience (Cam-CAN), University of Cambridge and MRC Cognition and Brain Sciences Unit, \\ Cambridge, UK
}

Background. Decades of research have investigated the impact of clinical depression on memory, which has revealed biases and in some cases impairments. However, little is understood about the effects of subclinical symptoms of depression on memory performance in the general population.

Methods. Here we report the effects of symptoms of depression on memory problems in a large population-derived cohort $(N=2544), 87 \%$ of whom reported at least one symptom of depression. Specifically, we investigate the impact of depressive symptoms on subjective memory complaints, objective memory performance on a standard neuropsychological task and, in a subsample $(n=288)$, objective memory in affective contexts.

Results. There was a dissociation between subjective and objective memory performance, with depressive symptoms showing a robust relationship with self-reports of memory complaints, even after adjusting for age, sex, general cognitive ability and symptoms of anxiety, but not with performance on the standardised measure of verbal memory. Contrary to our expectations, hippocampal volume (assessed in a subsample, $n=592$ ) did not account for significant variance in subjective memory, objective memory or depressive symptoms. Nonetheless, depressive symptoms were related to poorer memory for pictures presented in negative contexts, even after adjusting for memory for pictures in neutral contexts.

Conclusions. Thus the symptoms of depression, associated with subjective memory complaints, appear better assessed by memory performance in affective contexts, rather than standardised memory measures. We discuss the implications of these findings for understanding the impact of depressive symptoms on memory functioning in the general population.

Received 15 December 2016; Revised 5 May 2017; Accepted 9 May 2017; First published online 19 June 2017

Key words: Depression, emotion, emotional memory, memory, self-reported memory complaints.

\section{Introduction}

Mood fluctuates throughout the day as well as the lifespan, though overall most individuals feel 'fine' most of the time (Taquet et al. 2016). At one time or another, however, virtually everyone will experience low mood that is significant enough to endorse one or more symptoms of depression. In $24 \%$ of the cases, this will be severe enough to meet diagnostic criteria for major depressive disorder (Kessler et al. 2005; Kessler \& Bromet, 2013). Much needed research has been dedicated to the affective, cognitive and neurobiological correlates of these major depressive episodes (Davidson et al. 2002; Gotlib \& Joormann, 2010; Menon, 2011). Less

\footnotetext{
* Address for correspondence: S. Schweizer, MRC Cognition and Brain Sciences Unit, 15 Chaucer Road, Cambridge CB2 7EF, UK.

(Email: susanne.schweizer@mrc-cbu.cam.ac.uk)
}

however is known about the cognitive effects of depressive symptoms within the subclinical range commonly experienced by the general population. Here we explore the impact of depressive symptoms on memory, as experienced by a population cohort of over 2500 adults that was specifically selected for being currently free of neuropsychiatric disorders (the Cambridge Centre for Ageing and Neuroscience (Cam-CAN) cohort; http:// www.cam-can.org). Importantly, these individuals were tested on a range of memory measures including subjective memory complaints, performance on a standardized measure of memory, and performance (in a subset of the cohort) on a task specifically designed to assess memory in affective contexts.

The findings from the clinical literature suggest the ability to recall relevant information over time is reduced in individuals who experience depression (Burt et al. 1995; Rock et al. 2014). One possible

This is an Open Access article, distributed under the terms of the Creative Commons Attribution licence (http://creativecommons.org/licenses/by/4.0/), which permits unrestricted re-use, distribution, and reproduction in any medium, provided the original work is properly cited. 
neurobiological mechanism for these memory problems is prolonged exposure to elevated levels of corticosteroids, owing to the heightened psychological stress experienced in a depressive episode (Lamers et al. 2013; Baumeister et al. 2014; Hammen, 2015). The animal literature shows robust, well-replicated associations between stress exposure, levels of corticosteroids and memory performance, specifically through the neurodegenerative effect of corticosteroids on the hippocampus (for a review see: Kim \& Diamond, 2002), which is critical to the consolidation of information into long-term memory (Bird \& Burgess, 2008). Studies in human depression yield more equivocal results, though evidence generally supports the theory of volumetric shrinkage of the hippocampal complex in individuals suffering from depression (MacQueen \& Frodl, 2011; Fried \& Kievit, 2015). However, the field faces methodological challenges, some of which are inherent to the population under investigation. For example, comparing brain abnormalities across studies entails comparing across subtypes of depression, different levels of chronicity, environmental factors and variations in exposure to psychotropic medication (Fried et al. 2014). Moreover, little is known about how the effects of clinical depression on memory relate to the effects of (subclinical) symptoms of depression experienced in the general population. Building on the clinical literature, the present study aims to address this gap. We predicted that symptoms of depression experienced in the general population would be related to memory impairments (as assessed by a standardized measure of memory), and that this relationship would be associated with smaller hippocampal volumes.

The association between depressive symptoms and subjective memory complaints has been the focus of considerable previous research, especially in late adulthood (Jorm et al. 2001; Minett et al. 2008; Kim et al. 2013; Crumley et al. 2014; Yates et al. 2015). Research into the predictive utility of subjective memory complaints for objective memory performance and dementia diagnoses in older adults has revealed that subjective memory complaints may be better accounted for by individuals' levels of depressive symptomatology than their actual memory performance (e.g. Schofield et al. 1997; Reid \& MacLullich, 2006; Hülür et al. 2014; Yates et al. 2017). In line with these findings, we predicted that subjective memory complaints would increase as a function of symptoms of depression. Negative interpretative biases observed in those with clinical levels of depression, which increase as a function of symptoms of depression in non-clinical populations, may partially account for this finding (Mathews \& MacLeod, 2005; Beck, 2008). Alternatively the association between self-reported symptoms of depression and self-reported memory problems may simply reflect a response tendency on measures assessing neuropsychiatric health complaints. The current sample allowed a direct test of the latter hypothesis, by using the Hospital Anxiety and Depression Scale (HADS; Zigmond \& Snaith, 1983) to assess symptoms of depression. The two subscales of the HADS assess symptoms of anxiety and depression, respectively. If increased memory complaints reflect a simple response tendency, then symptoms of depression and anxiety should show the same association with subjective memory complaints. In contrast, if the increase in self-reported memory complaints is specific to depressive symptomatology, then subjective memory complaints should be more reliably associated with symptoms of depression than symptoms of anxiety.

Previous work additionally suggests that altered cognitive and affective processing in depression are associated with other changes in memory performance. For example, depressed individuals exhibit a mood-congruency bias, which makes them able to recall more negative memoranda compared with nondepressed individuals (Elliott et al. 2002). Another memory phenomenon observed in individuals with depression is that their autobiographical memories lack specificity (i.e. depressed individuals memories are typically overgeneral; Dalgleish \& Werner-Seidler, 2014; Dritschel et al. 2014). These deviations from typical memory performance suggest an abnormality in basic memory operation and/or in the processing of affective information. Research on memory for affective stimuli and events more broadly shows that compared with neutral, affective information is better remembered (LaBar \& Cabeza, 2006). What remains under-researched is the effect of affective context on memory performance. Henson et al. (2016) have recently shown that, in the same Cam-CAN cohort as is studied here, recognition memory for neutral objects varied as a function of the affective valence (negative, positive or neutral) of the background context against which those objects were originally presented. The increased affective significance (cf. Pessoa, 2009) of negative information to individuals who currently experience symptoms of depression is likely to attract attentional resources towards negative backgrounds and away from neutral objects superimposed on those backgrounds, thereby impairing the encoding into memory of those objects. For this reason, memory for information presented in affective contexts may be more sensitive to the influence of subclinical depressive symptoms than the more commonly used, affectneutral measures of memory.

In summary, the present study investigated the hypotheses that depressive symptoms are related to 
more subjective memory complaints (Hypothesis 1a) and worse objective memory performance (Hypothesis 1b). This first pair of hypotheses was investigated in all individuals from the Cam-CAN cohort who completed all measures of interest during an interview assessment in participants' homes $(N=2544)$. The study further explored whether the relationship between memory performance and depressive symptoms is related to reductions in hippocampal volumes (Hypothesis 2). This was investigated in a subsample $(n=592)$ for whom volumetric data of the hippocampus were available from a more extensive neurocognitive assessment including the acquisition of T1- and T2-weighted MRI scans. The third prediction was that self-reported symptoms of depression would be more strongly related to a measure of memory in negative contexts compared with a standard measure of memory (Hypothesis 3). This hypothesis was investigated in a second subsample $(n=288)$ that completed a more specialized memory task.

The nature of the study's sample also allowed for a number of additional explorations: first, as outlined above, we tested whether H1a was specific to symptoms of depression. That is, whether the relationship between depressive symptoms and self-reported memory complaints reflected a general response tendency towards reporting more neuropsychiatric complaints and would therefore show the same relationship with symptoms of anxiety. Next, given the evidence suggesting that memory problems related to depressive symptoms may be a function of general impairments in cognitive ability observed in individuals experiencing symptoms of depression (Fossati et al. 2002; Elderkin-Thompson et al. 2007) the study investigated whether symptoms of depression remained significantly related to the various types of memory after controlling for general cognitive ability. Third, the study explored whether the relationships in hypotheses 1-3 would remain after accounting for variations in memory, hippocampal volume and depressive symptoms attributable to age (Jeste et al. 2013; Sutin et al. 2013; Schaakxs et al. 2017). And finally, because women tend to show better verbal recall performance and more symptoms of depression, we investigated whether sex differences contribute to the relationships predicted in hypotheses 1-3 (Piccinelli \& Wilkinson, 2000; Andreano \& Cahill, 2009).

\section{Methods}

\section{Participants}

The full sample included 2544 individuals from the CC3000 Cam-CAN sample (Shafto et al. 2014). These participants ( $95 \%$ of the total Cam-CAN sample) were included because they had completed all measures pertaining to our first hypothesis. Structural imaging data was available for 592 participants from our overall sample. Hypothesis 2 was tested on this subsample. Finally, 288 participants from the total sample completed the valenced memory task and were included in the investigation of our third hypothesis. See online Supplementary Table S1 for participant characteristics.

\section{Measures \\ Depressive symptoms}

Symptoms of depression were assessed with the depression subscale of the HADS (Zigmond \& Snaith, 1983). The subscale consists of seven items for which participants indicate how frequently they have felt them over the past week on a scale form ' 0 ' $=$ 'Not at all' to ' 3 ' = 'Most of the time'. The scales have been well validated for use in the general population (Olssøn et al. 2005). In the current sample Cronbach's $\alpha$ was acceptable-good, $a=0.79$ and similarly McDonalds' $\Omega$ hierarchical was 0.71 .

\section{Objective memory}

Objective memory was assessed with a standard measure of memory, the delayed recall of a story taken from the Wechsler Memory Scale Third UK edition (Wechsler, 1997).

\section{Subjective memory}

Participants were simply asked whether they experienced any memory problems or not: 'Do you feel you have problems with your memory? Yes/No.'.

\section{Valenced memory}

Memory for objects in positively and negatively valenced as well as valence neutral contexts was assessed with a newly designed measure (Henson et al. 2016). The task consisted of a study and a test phase. The study phase was divided into two $10 \mathrm{~min}$ blocks with a short break in between. In each block participants were presented with 60 background images selected from the International Affective Picture System (Lang et al. 2008) that appeared on the screen for $2 \mathrm{~s}$ before an object was superimposed on the background image. The object and background stayed on the screen for $7.5 \mathrm{~s}$. Participants were asked to press the button as soon as they had come up with a story to help them link the object and background together. They were asked to keep elaborating on that story until the object and background 
disappeared. There was a 0.5 interval second before the next trial started.

Participants were advised that some images would be pleasant and others unpleasant, but they were not informed that their memory for the items and their background would be tested later. After participants completed the second block they were given a $10 \mathrm{~min}$ break before the test phase. In the test phase they saw 160 trials that were split into four 20 min blocks. The trials included 120 studied objects and 40 new objects. Each test trial first presented participants with a masked (pixel noise) picture of an object and participants had to name the object or respond 'I don't know' before pressing the key to reveal the object. Memory for the object was then tested by asking participants whether or not the object had been presented in the study phase. Participants then indicated how confident they were of their response by pressing one of four keys: 'sure new', 'think new', 'sure studied' or 'think studied'. For trials on which participants indicated 'studied sure' or 'studied think' their associative memory was tested. That is, participants were asked to say out loud whether the object had been presented over a positive, neutral or negative background or to respond 'I don't know', if they could not remember the valence of the background. Finally, they were asked to describe the background image. The priming, associative memory and qualitative data are not reported as part of this study.

Memory accuracy was computed as the $d^{\prime}$ measure of discriminability (Green \& Swets, 1966): $d^{\prime}=\Phi^{-1}$ $(\mathrm{pH})-\Phi^{-1}$ (pFA). The $\mathrm{pH}$ denotes the proportion of hits, pFA the proportion of false alarms and $\Phi^{-1}$ the inverse cumulative distribution function of the Normal distribution ( $d^{\prime}=0$ for chance performance; extreme values of 0 or 1 for $d^{\prime}$ were adjusted using a log-linear approach).

\section{General cognitive functioning}

In the overall cohort, cognitive ability was assessed with the Addenbrooke's Cognitive Examination Revised assessment (ACE-R; Mioshi et al. 2006). The screening measure was devised to detect signs of dementia and cognitive impairment assessing five domains of cognitive functioning: orientation/attention, memory, verbal fluency, language and visuospatial ability. The memory domain assess both immediate and delayed recall. As with our assessment of objective memory, participants had to recall verbal information after a delay interval. We therefore used the ACE-R sum score of all domains except memory. For the neuroimaging and affective subsamples, the ACE-R was not an informative test of cognitive ability due to participants scoring at ceiling. We therefore included the Cattell's culture-free test of intelligence (Cattell, 1971), which was available in both subsamples (not the overall cohort). The test requires participants to complete complex pattern matrices, and has previously shown strong associations with behavioural and neural domains within the Cam-CAN cohort (Kievit et al. 2014).

\section{Structural MRI}

Grey matter was estimated from the combined segmentation of $1 \mathrm{~mm}^{3}$, T1- and T2-weighted MR images, followed by diffeomorphic registration of grey-matter segments from all participants in Stage 2 of the Cam-CAN study in order to create a sample-specific template. This template was then transformed into Montreal Neurological Institute (MNI) space, and every participant's gray-matter image resliced to the same space, while being modulated by the warping entailed. These stages were done in SPM12 (http:// www.fil.ion.ucl.ac.uk/spm). For details of the MRI sequences, see (Shafto et al. 2014) for further details of the MRI preprocessing, see (Taylor et al. 2017). We estimated the mean grey matter volume across voxels within the hippocampus, by modulating the grey matter density in each voxel by the Jacobean of the warps used to transform to MNI space within the left and right Hippocampal ROIs (regions of interest) from the Harvard-Oxford atlas (http://fsl.fmrib.ox.ac.uk/fsl/ fslwiki/Atlases), as is standard in many previous studies.

\section{Statistical analyses}

Given the non-normal distribution of depressive symptoms in the cohort (Fig. 1), we ran all analyses as nonparametric tests. More specifically, we entered the HADS-scores into a non-parametric logistic regression analyses based on ranks for the dichotomous outcome (i.e. subjective memory complaints) and non-parametric regression for the continuous outcomes (i.e. standard and affective memory measures). Given the directionality of our hypotheses, all significance testing of our a priori hypotheses was one-tailed, whereas significance level for all exploratory tests was two-tailed.

\section{Procedure}

After providing informed written consent, participants completed the 'Stage 1 - Interview' of the Cam-CAN study including computerised health and lifestyle questionnaires as well as a core cognitive assessment (Shafto et al. 2014). The subset of measures included in the present study are described below. A subset of the sample (subsamples selected to investigate hypotheses 2 and 3, see above) were included in the 'Stage 2 - Core Cognitive 


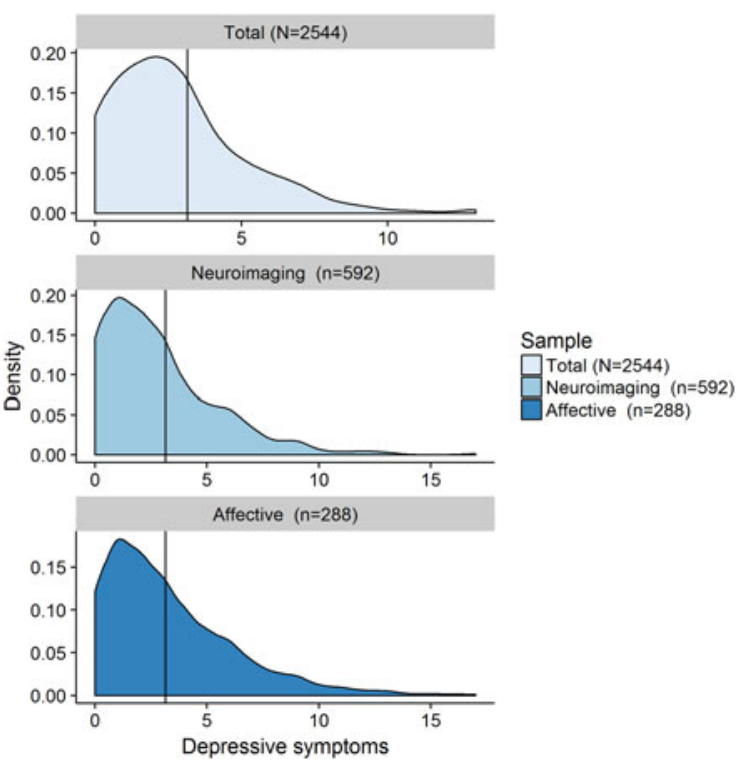

Fig. 1. Neuroimaging cohort=individuals from the overall cohort for whom structural neuroimaging data is available; Affective cohort $=$ individuals from the overall cohort who completed the valenced memory measure; $N / n=$ number of participants; Depressive symptoms = number of self-reported symptoms of depression on the Hospital Anxiety and Depression Scale (HADS) depression subscale (range 0-21, Zigmond \& Snaith, 1983).

Neuroscience' phase of the Cam-CAN study. As part of the second stage of the study, participants completed a series of cognitive task across three sessions. One of these sessions also included core structural and functional MRI measures. This second stage also included the valenced memory task. Given the time constraints across multiple sessions, the Cam-CAN protocol included a subset of tasks, including the valenced memory tasks, administered to only a randomized subset (50\%) of participants. This study complied with the Helsinki Declaration, and was approved by the Cambridgeshire 2 Research Ethics Committee (reference: 10/H0308/50).

\section{Results}

Figure 1 shows the distribution of scores from the depression subscale of the HADS, while online Supplementary Table S1 provides a comprehensive overview of the characteristics of all three samples. We found that $87 \%(n=2211)$ of participants reported at least one symptom of depression, and over $91 \%$ scored a total of seven points or less on the depression subscale (Zigmond \& Snaith, 1983), which is below the commonly reported clinical cutoff of eight points for this subscale (Bjelland et al. 2002). This shows that symptoms of depression are common in the general population, but typically do not reach clinically significant levels ${ }^{+}$.

In the full cohort $(N=2544)$, more symptoms of depression were related to more frequent self-report of memory complaints, $\beta=6.99^{-4}$, s.D. $=5.94^{-5},[95 \%$ confidence interval (CI) $5.83^{-4}-8.16^{-4}$ ], $z=11.76, p \leqslant$ $0.001, R_{\text {Nagelkerke }}^{2}=0.08(\mathrm{H} 1 \mathrm{a})$ and poorer performance on a standardized measure of memory, $\beta=-1.00^{-3}$, S.D. $=1.32^{-4},\left(95 \%\right.$ CI $-1.00^{-3}$ to $\left.-8.58^{-4}\right), t(2542)=$ $-8.44, p \leqslant 0.001, R^{2}=0.03$ (H1b, Fig. 2a). However, only the relationship between depressive symptoms and subjective memory survived adjustment for age, cognitive ability ${ }^{2}$ and $\mathrm{sex}^{3}, \beta=5.19^{-4}$, s.D. $=6.37^{-5}$, $\left(95 \%\right.$ CI $\left.3.94^{-4}-6.44^{-4}\right), z=8.15, p \leqslant 0.001, R_{\text {Nagelkerke }}^{2}$ $=0.20$; the same was not true for the relationship with standardized memory performance, $\beta=-1.31^{-4}$, S.D. $=1.14^{-4},\left(95 \%\right.$ CI $-1.00^{-3}$ to $\left.-8.58^{-4}\right), t(2539)=$ $-1.15, p=0.125, R^{2}=0.33, R_{\mathrm{adj}}^{2}=0.33$ (Fig. $2 b$ ). The absence of a relationship was confirmed with Bayesian analysis (JASP Team, 2016), $\mathrm{BF}_{01}=10.85$. Moreover, exploratory analyses showed that the relationship between symptoms of depression and subjective memory complaints did not appear to be due to individuals who suffer from symptoms of depression simply reporting more neuropsychiatric health complaints. That is, while symptoms of anxiety were related to subjective memory complaints, $\beta=2.35^{-4}$, S.D. $=5.63^{-5},\left(95 \%\right.$ CI $\left.1.24^{-4}-3.45^{-4}\right), z=4.16, p \leqslant 0.001$, $R_{\text {Nagelkerke }}^{2}=0.01$, the relationship was no longer significant when accounting for depressive symptoms in the same analyses, $\beta=-5.63^{-5}, \quad$ S.D. $=6.30^{-5}, \quad(95 \%$ CI $-1.80^{-4}$ to $\left.6.71^{-4}\right), z=-0.89, p=0.372, R_{\text {Nagelkerke }}^{2}=$ 0.00 , whereas the relationship between depression and subjective memory complaints remained significant after adjusting for symptoms of anxiety, $\beta=$ $7.23^{-4}$, s.D. $=6.52^{-5},\left(95 \%\right.$ CI $\left.5.96^{-4}-8.51^{-4}\right), z=11.09$, $p \leqslant 0.001, R_{\text {Nagelkerke }}^{2}=0.08$.

In the neuroimaging cohort $(n=592)$, multiple regression including total intracranial volume (TIV) as a covariate showed that hippocampal volume was related to both subjective memory, $\beta=-4.64$, S.D. $=$ 1.71, (95\% CI -8.02 to -1.23$), z=-2.71, p=0.004$, $R_{\text {Nagelkerke }}^{2}=0.02$, and the standardized objective measure, $\beta=17.94$, S.D. $=3.29$, (95\% CI 11.47-24.41), $t(589)=$ $5.45, p \leqslant 0.001, R^{2}=0.05, R_{\text {adj }}^{2}=0.04$ (Fig. 3a). However, neither the relationship between hippocampal volume

\footnotetext{
+ The notes appear after the main text

${ }^{1}$ We also ran all analyses excluding the $9 \%$ of participants who scored above the clinical cutoff $(\geqslant 8)$, and found pattern of associations virtually unchanged.

${ }^{2}$ Measured with the ACE-R sum score excluding the memory subscale.

${ }^{3}$ See online Supplementary Table S2 for the association between the memory measures and the covariates: age, cognitive ability and sex.
} 


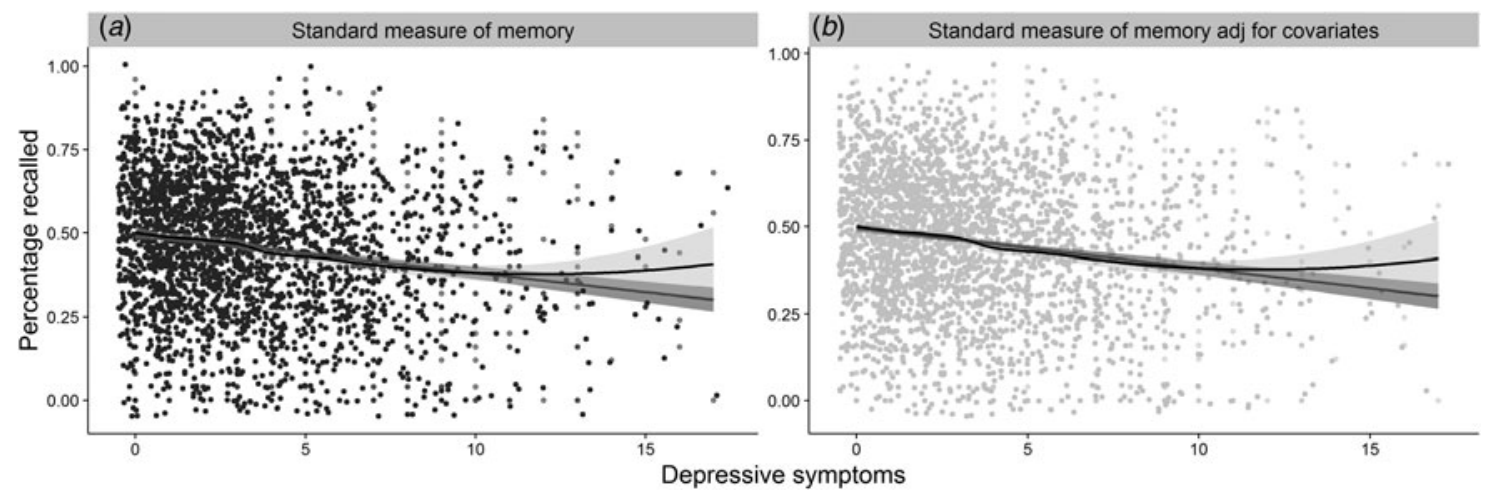

Fig. 2. The figure represents the relationships between: (a) depressive symptoms and performance on the standard measure of objective memory; (b) 2a, after adjustment for age, cognitive ability and sex; jitter was added to the distribution for illustration purposes.

and subjective memory complaints, $\beta=0.43$, S.D. $=2.00$, (95\% CI -3.50 to -4.36$), z=0.21, p=0.416, R_{\text {Nagelkerke }}^{2}=$ 0.07 , nor relationship between hippocampal volume and objective memory $\beta=0.22$, s.D. $=3.40, \quad(95 \% \mathrm{CI}$ -6.45 to 6.90$), t(586)=0.07, p=0.474, R^{2}=0.25, R_{\text {adj }}^{2}=$ 0.24 , survived adjustment for age, cognitive ability 4 and sex. Contrary to our expectations, there was no significant association between depressive symptoms and hippocampal volume, $\beta=-2.57$, s.D. $=2.01$, $(95 \%$ CI -6.51 to 1.38$), t(589)=1.28, p=0.100, R^{2}=0.00$ (Fig. $3 b$ ), which was again supported by a Bayes factor of 6.87 in favour of the null hypothesis. Unsurprisingly therefore, the relationships between depressive symptoms and both subjective $\beta=3.00^{-1}$, s.D. $=5.59^{-4},(95 \%$ CI $\left.2.00^{-1}-4.00^{-1}\right), z=5.04, p \leqslant 0.001, R_{\text {Nagelkerke }}^{2}=0.08$ and objective $\beta=-2.00^{-1}$, S.D. $=1.00^{-1}, \quad(95 \% \quad \mathrm{CI}$ $-4.00^{-1}$ to $\left.1.03^{-4}\right), t(588)=-1.86, p=0.032, R^{2}=0.05$, $R_{\text {adj }}^{2}=0.05$ memory remained significant after adjusting for hippocampal volume (and TIV). That is, there was no support for the hypothesis that hippocampal volumes account in part for the relationship between depressive symptoms and memory performance in this non-clinical population $(\mathrm{H} 2)$.

In the cohort that completed the measure of memory in affective contexts $(n=288)$, we investigated whether depressive symptoms showed a differential association with memory performance in affective contexts compared with a standardized measure of memory (H3). As in the overall sample, the relationship between depressive symptoms and performance on a standardized memory measure, $\beta=-5.00^{-1}$, S.D. $=3.00^{-1}$, $\left(95 \% \mathrm{CI}-1.10^{-1}\right.$ to $\left.6.91^{-4}\right), t(286)=-1.78, p=0.043$, $R^{2}=0.01$, did not survive adjustment for age, cognitive ability $^{5}$, and sex, $\beta=-2.00^{-1}$, S.D. $=3.00^{-1}$, (95\% CI

\footnotetext{
${ }^{4}$ Measured with the Cattel's culture-free test of intelligence.

${ }^{5}$ Measured with the Cattel's culture-free test of intelligence.
}

$-7.00^{-1}$ to $\left.3.00^{-1}\right), t(283)=-0.66, p=0.509, R^{2}=0.23$, $R_{\text {adj }}^{2}=0.21, \mathrm{BF}_{01}=4.69$. Symptoms of depression were, however, significantly related to poorer memory performance in negative, $\beta=-4.00^{-2}$, S.D. $=1.00^{-2},(95 \%$ $\mathrm{CI}-6.00^{-2}$ to $\left.-1.00^{-2}\right), t(286)=-3.48, p \leqslant 0.001, R^{2}$ $=0.04$ and positive $\beta=-2.00^{-2}$, S.D. $=1.00^{-2},(95 \% \mathrm{CI}$ $-5.00^{-2}$ to $\left.-6.39^{-5}\right), t(286)=-2.52, p=0.006, R^{2}=$ 0.02 contexts (Fig. 4). However, when adjusting for performance in neutral contexts only the relationship between depressive symptoms and negative context remained significant, $\beta=-1.45^{-4}$, S.D. $=5.93^{-5}, \quad(95 \%$ CI $-2.61^{-4}$ to $\left.-2.79^{-5}\right), t(285)=-2.44, p=0.015, R^{2}=$ $0.76, R_{\text {adj }}^{2}=0.76$, even after adjusting for the same covariates, $\beta=-1.38^{-4}$, S.D. $=5.78^{-5},\left(95 \% \mathrm{CI}-2.51^{-4}\right.$ to $\left.-2.40^{-5}\right), \quad t(285)=-2.38, \quad p=0.018, \quad R^{2}=0.78, \quad R_{\text {adj }}^{2}=$ 0.78 ., but depressive symptoms were not related to memory in positive contexts after adjusting for performance in neutral contexts, $\beta=-3.22^{-5}$, S.D. $=5.85^{-5}$, $\left(95 \% \mathrm{CI}-1.47^{-4}\right.$ to $\left.8.32^{-5}\right), t(285)=-0.55, p=0.584$, $R^{2}=0.76, R_{\text {adj }}^{2}=0.75$ and covariates, $\beta=-2.15^{-5}$, s.D. $=$ $5.76^{-5}$, (95\% CI $-1.00^{-3}$ to $\left.9.19^{-5}\right), t(282)=-0.37$, $p=0.709, R^{2}=0.77, R_{\mathrm{adj}}^{2}=0.76$. Moreover, when controlling for memory in positive contexts, depressive symptoms remained a significant predictor of memory in negative contexts, $\beta=-1.46^{-4}$, S.D. $=5.52^{-5},(95 \% \mathrm{CI}$ $-2.54^{-4}$ to $\left.-3.75^{-5}\right), t(285)=-2.58, p=0.010, R^{2}=$ $0.79, R_{\text {adj }}^{2}=0.79$, even after adjusting for the covariates, $\beta=-1.39^{-4}$, s.D. $=5.39^{-5},\left(95 \% \mathrm{CI}-2.45^{-4}\right.$ to $\left.-3.29^{-5}\right)$, $t(285)=-2.58, p=0.009, R^{2}=0.81, R_{\mathrm{adj}}^{2}=0.81$.

Furthermore, when directly comparing the size of the relationship of depressive symptoms with memory for objects in negative contexts with that for objects in positive contexts, the former was significantly higher, Williams' $t(285)=3.07, p=0.002$. There was a marginal relationship between symptoms of depression and memory for objects presented in negative contexts to be stronger than their relationship with memory on a standardized measure of memory, Williams' $t$ (285) $=-1.70, p=0.045$ (H3). 

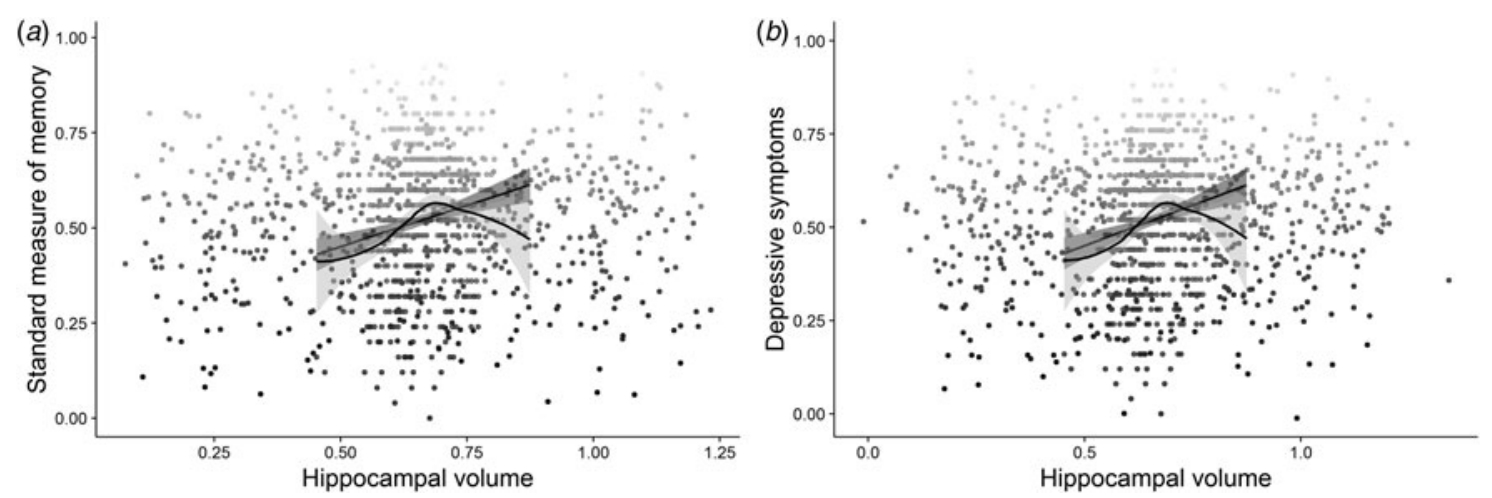

Fig. 3. The figure represents the relationships between: (a) hippocampal volume and performance on the standard measure of memory; (b) hippocampal volume and symptoms of depression; jitter was added to the distribution for illustration purposes.

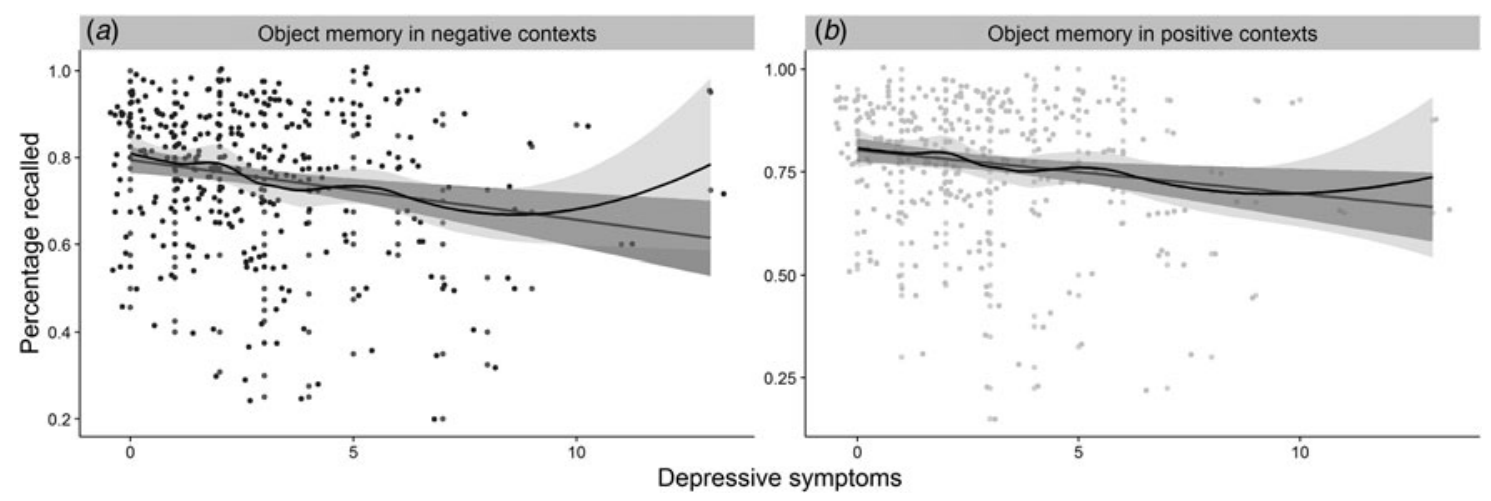

Fig. 4. The figure represents the relationships between: (a) depressive symptoms and memory for objects presented in negative contexts; $(b)$ depressive symptoms and memory for objects presented in positive contexts; jitter was added to the distribution for illustration purposes.

Finally, we explored whether self-reported history of depression moderated any of the associations between current symptoms of depression and memory performance (H1), memory performance accounting for hippocampal volume (H2) or memory in affective contexts (H3). Self-reported history of depression that required treatment did not moderate any of the associations significantly, $t s<1, p s \geqslant 0.40$.

\section{Discussion}

The present study examined the memory correlates of depressive symptoms in a large, population-derived cohort. First, we showed that depressive symptoms were related to self-reported memory problems, even after controlling for variations in age, cognitive ability and sex. Moreover, the relationship was not simply due to individuals' tendency to report more mental health problems, as the relationship between depressive symptoms and subjective memory remained after adjusting for symptoms of anxiety. One possibility is that the association between symptoms of depression and subjective memory reflects a negative interpretative bias. This notion, known as 'depressive realism', suggests that individuals who report symptoms of depression show less positivity bias (Mezulis et al. 2004; Watson et al. 2007). Future research should therefore investigate whether other types of selfreported cognitive functioning problems, for example attentional control problems (Derryberry \& Reed, 2002), are also selectively associated with symptoms of depression but not other measures of mental health functioning.

A second set of findings showed that depressive symptoms were also related to performance on a standardized test of memory, but in this case, we could not rule out the possibility that this relationship was due to variations in memory as a function of age, cognitive ability and/or sex (which were all significantly related to objective memory; see online Supplementary Table S2). In line with Fried \& Kievit (2015), we also found no evidence for a significant relationship between depressive symptoms and hippocampal volume in this non-clinical cohort. Future research could investigate alternative sources of brain alterations 
associated with commonly experienced symptoms of depression (Hamilton et al. 2008). Alternatively, the lack of an association between depressive symptoms and hippocampal volume may be because the current sample was a non-clinical sample (i.e. participants reported no functional impairment form their depressive symptoms). To date, hippocampal volume has been studied in the context of clinical depression, and its association with subclinical symptoms of depression remains under researched. There is some evidence pointing towards possible gender differences, with men showing an association between subclinical symptoms of depression and hippocampal volumes but not women (Hayakawa et al. 2013; Samplin et al. 2013; Spalletta et al. 2014) ${ }^{6}$.

Finally, a third investigation showed that, while performance on a standard memory test may be unaffected in individuals experiencing subclinical symptoms of depression, objective memory impairments are found when the memoranda are encountered in negatively valenced settings. More specifically, depressive symptoms were related to worse recognition memory for visual objects presented against negative backgrounds, even when adjusting for age, cognitive ability and sex. Importantly, this relationship remained even when further adjusting for recognition memory for the same types of objects presented against neutral backgrounds. This suggests that the relationship was specific to the valenced context, rather than differences between the visual object recognition memory test and the standardized verbal recall test, in terms of, for example, the nature of the memoranda or retrieval demands. Furthermore, the relationship between depressive symptoms and recognition memory for objects in positive contexts was no longer significant after the same adjustment for memory in neutral contexts, and the size of the relationship between depressive symptoms and recognition memory for objects in negative contexts was significantly greater than that between depressive symptoms and memory in positive contexts. In other words, the sensitivity of memory to depressive symptoms was selective to memory in negative contexts.

The implication of this finding is that measures of memory in negatively valenced contexts (e.g. Henson et al. 2016) may be particularly sensitive to subtle differences in memory performance caused by current affective state. Importantly, the study demonstrates

\footnotetext{
${ }^{6}$ In the light of these preliminary findings we explored the potentially moderating effect of sex on the association between hippocampal volume and depressive symptoms. In the current sample there was no evidence for a moderating effect of sex (see online Supplementary Material).
}

that even subclinical depressive symptoms appear to have an impact on both self-perceived and objective measures of memory functioning. Again it remains an open question as to whether the impact of subclinical depressive symptoms in the general population is limited to memory performance in negative contexts, or whether this extends to other types of higher cognitive functions in negatively laden environments.

When considering the implications of the findings from this large-scale population-based study, a few limitations should be noted. First, subjective memory impairments were only assessed with a single item, which may not be a sensitive measure of subjective concerns about memory functioning. Moreover, inferences about subclinical levels of depression and self-reported history of clinical episodes of major depression need to be drawn with caution because they were assessed through a self-report questionnaire and retrospective recall, respectively. These assessments may differ from clinician-rated levels of past and present depressive symptomatology in the current sample.

Future research should explore whether the association between depressive symptoms and the different memory measures observed in the current sample will replicate in currently depressed individuals, or whether they exhibit different memory profiles. Given the impairment in autobiographical memory specificity that is characteristic of individuals in a major depressive episode, the relation between performance on the affective memory measure and the specificity of autobiographical memory should be assessed in healthy and currently depressed individuals. Future research should also investigate whether the strength of the association between individuals' memory performance in negative contexts and their symptoms of depression has predictive utility for the development of more severe clinical forms of depressive disorders. That is, whether memory for neutral information in negative context fits within a larger pattern of cognitive vulnerabilities to depression (Gotlib \& Joormann, 2010).

In conclusion, these findings show that the frequency of self-reported memory problems increases as a function of subclinical depressive symptoms. However, depressive symptoms are not associated with memory performance on a standard objective memory measure, when controlling for age, general cognitive ability and sex. Rather, depressive symptoms are associated with poorer memory for objects presented in negative contexts. These results suggest that memory for objects presented in negative contexts may be particularly sensitive to the memory problems reported by those experiencing symptoms of depression. 


\section{Supplementary material}

The supplementary material for this article can be found at https://doi.org/10.1017/S0033291717001519.

\section{Acknowledgements}

The Cambridge Centre for Ageing and Neuroscience (Cam-CAN) research was supported by the Biotechnology and Biological Sciences Research Council (grant number BB/H008217/1). SS is supported by UK Medical Research Council Programme MC-A060-5PQ60; RNH and TE are supported by MC-A060-5PR10; RAK is supported by MC-A060-5PR60 and a Sir Henry Wellcome Trust Fellowship (grant number 107392/Z/15/ $\mathrm{Z}$ ). The authors are grateful to the Cam-CAN respondents and their primary care teams in Cambridge for their participation in this study. They also thank colleagues at the MRC Cognition and Brain Sciences Unit MEG and MRI facilities for their assistance. The Cam-CAN corporate author consists of the project principal personnel: Lorraine K Tyler, Carol Brayne, Edward T Bullmore, Andrew C Calder, Rhodri Cusack, Tim Dalgleish, John Duncan, Fiona E Matthews, William D Marslen-Wilson, James B Rowe, Meredith A Shafto; Research Associates: Karen Campbell, Teresa Cheung, Simon Davis, Linda Geerligs, Anna McCarrey, Abdur Mustafa, Darren Price, David Samu, Jason R Taylor, Matthias Treder, Kamen Tsvetanov, Janna van Belle, Nitin Williams; Research Assistants: Lauren Bates, Tina Emery, Sharon Erzinçlioglu, Andrew Gadie, Sofia Gerbase, Stanimira Georgieva, Claire Hanley, Beth Parkin, David Troy; Affiliated Personnel: Tibor Auer, Marta Correia, Lu Gao, Emma Green, Rafael Henriques; Research Interviewers: Jodie Allen, Gillian Amery, Liana Amunts, Anne Barcroft, Amanda Castle, Cheryl Dias, Jonathan Dowrick, Melissa Fair, Hayley Fisher, Anna Goulding, Adarsh Grewal, Geoff Hale, Andrew Hilton, Frances Johnson, Patricia Johnston, Thea Kavanagh-Williamson, Magdalena Kwasniewska, Alison McMinn, Kim Norman, Jessica Penrose, Fiona Roby, Diane Rowland, John Sargeant, Maggie Squire, Beth Stevens, Aldabra Stoddart, Cheryl Stone, Tracy Thompson, Ozlem Yazlik; and Administrative Staff: Dan Barnes, Marie Dixon, Jaya Hillman, Joanne Mitchell, Laura Villis.

\section{References}

Andreano JM, Cahill L (2009). Sex influences on the neurobiology of learning and memory. Learning \& Memory 16, 248-266.

Baumeister D, Lightman SL, Pariante CM (2014). The interface of stress and the HPA axis in behavioural phenotypes of mental illness. Current Topics in Behavioural Neuroscience 18, 13-24.
Beck AT (2008). The evolution of the cognitive model of depression and its neurobiological correlates. American Journal of Psychiatry 165, 969-977.

Bird CM, Burgess N (2008). The hippocampus and memory: insights from spatial processing. Nature Reviews Neuroscience 9, 182-194.

Bjelland I, Dahl AA, Haug TT, Neckelmann D (2002). The validity of the Hospital Anxiety and Depression Scale: an updated literature review. Journal of Psychosomatic Research 52, 69-77.

Burt DB, Zembar MJ, Niederehe G (1995). Depression and memory impairment: a meta-analysis of the association, its pattern, and specificity. Psychological Bulletin 117, 285-305.

Cattell RB (1971). Abilities: their Structure, Growth and Action. Houghton Mifflin: Boston, MA.

Crumley JJ, Stetler CA, Horhota M (2014). Examining the relationship between subjective and objective memory performance in older adults: a meta-analysis. Psychology and Aging 29, 250-263.

Dalgleish T, Werner-Seidler A (2014). Disruptions in autobiographical memory processing in depression and the emergence of memory therapeutics. Trends in Cognitive Sciences 18, 596-604.

Davidson RJ, Pizzagalli D, Nitschke JB, Putnam K (2002). Depression: perspectives from affective neuroscience. Annual Review of Psychology 53, 545-574.

Derryberry D, Reed MA (2002). Anxiety-related attentional biases and their regulation by attentional control. Journal of Abnormal Psychology 111, 225-236.

Dritschel B, Beltsos S, McClintock SM (2014). An 'alternating instructions' version of the Autobiographical Memory Test for assessing autobiographical memory specificity in non-clinical populations. Memory 22, 881-889.

Elderkin-Thompson V, Mintz J, Haroon E, Lavretsky H, Kumar A (2007). Executive dysfunction and memory in older patients with major and minor depression. Archives of Clinical Neuropsychology 22, 261-270.

Elliott R, Rubinsztein JS, Sahakian BJ, Dolan RJ (2002). The neural basis of mood-congruent processing biases in depression. Archives of General Psychiatry 59, 597-604.

Fossati P, Coyette F, Ergis A-M, Allilaire J-F (2002). Influence of age and executive functioning on verbal memory of inpatients with depression. Journal of Affective Disorders 68, 261-271.

Fried EI, Kievit RA (2015). The volumes of subcortical regions in depressed and healthy individuals are strikingly similar: a reinterpretation of the results by Schmaal et al. Molecular Psychiatry 21, 724-725.

Fried EI, Tuerlinckx F, Borsboom D (2014). Mental health: more than neurobiology. Nature 508, 458-458.

Gotlib IH, Joormann J (2010). Cognition and depression: current status and future directions. Annual Review of Clinical Psychology 6, 285-312.

Green DM, Swets JA (1966). Signal Detection and Psychophysics. Wiley: New York, NY.

Hamilton JP, Siemer M, Gotlib IH (2008). Amygdala volume in major depressive disorder: a meta-analysis of magnetic resonance imaging studies. Molecular Psychiatry 13, 993-1000. 
Hammen CL (2015). Stress and depression: old questions, new approaches. Current Opinion in Psychology 4, 80-85.

Hayakawa YK, Sasaki H, Takao H, Mori H, Hayashi N, Kunimatsu A, Aoki S, Ohtomo K (2013). Structural brain abnormalities in women with subclinical depression, as revealed by voxel-based morphometry and diffusion tensor imaging. Journal of Affective Disorders 144, 263-268.

Henson RN, Campbell KL, Davis SW, Taylor JR, Emery T, Erzinclioglu S, Cam-CAN, Kievit RA (2016). Multiple determinants of lifespan memory differences. Scientific Reports 6, 32527.

Hülür G, Hertzog C, Pearman A, Ram N, Gerstorf D (2014). Longitudinal associations of subjective memory with memory performance and depressive symptoms: between-person and within-person perspectives. Psychology and Aging 29, 814-827.

JASP Team (2016). JASP.

Jeste DV, Savla GN, Thompson WK, Vahia IV, Glorioso DK, Martin AS, Palmer BW, Rock D, Golshan S, Kraemer HC, Depp CA (2013). Association between older age and more successful aging: critical role of resilience and depression. American Journal of Psychiatry 170, 188-196.

Jorm AF, Christensen H, Korten AE, Jacomb PA, Henderson AS (2001). Memory complaints as a precursor of memory impairment in older people: a longitudinal analysis over 7-8 years. Psychological Medicine 31, 441.

Kessler RC, Berglund P, Borges G, Nock M, Wang PS (2005). Trends in suicide ideation, plans, gestures, and attempts in the United States, 1990-1992 to 2001-2003. JAMA: Journal of the American Medical Association 293, 2487.

Kessler RC, Bromet EJ (2013). The epidemiology of depression across cultures. Annual Review of Public Health 34, 119-138. doi: 10.1146/annurev-publhealth-031912114409.

Kievit RA, Davis SW, Mitchell DJ, Taylor JR, Duncan J, Cam-CAN, Henson (2014). Distinct aspects of frontal lobe structure mediate age-related differences in fluid intelligence and multitasking. Nature Communications 5, 5658.

Kim JJ, Diamond DM (2002). The stressed hippocampus, synaptic plasticity and lost memories. Nature Reviews Neuroscience 3, 453-462.

Kim M-J, Seo SW, Kim GH, Kim ST, Lee J-M, Qiu A, Na DL (2013). Less depressive symptoms are associated with smaller hippocampus in subjective memory impairment. Archives of Gerontology and Geriatrics 57, 110-115.

LaBar KS, Cabeza R (2006). Cognitive neuroscience of emotional memory. Nature Reviews Neuroscience 7, 54-64.

Lamers F, Vogelzangs N, Merikangas KR, De Jonge P, Beekman ATF, Penninx B (2013). Evidence for a differential role of HPA-axis function, inflammation and metabolic syndrome in melancholic versus atypical depression. Molecular Psychiatry 18, 692-699.

Lang PJ, Bradley MM, Cuthbert BN (2008). International Affective Picture System (IAPS): Affective Ratings of Pictures and Instruction Manual (Technical Report). University of Florida, NIMH Center for the Study of Emotion and Attention: Gainesville, FL.

MacQueen G, Frodl T (2011). The hippocampus in major depression: evidence for the convergence of the bench and bedside in psychiatric research? Molecular Psychiatry 16, 252-264.

Mathews A, MacLeod C (2005). Cognitive vulnerability to emotional disorders. Annual Review of Clinical Psychology 1, 167-195.

Menon V (2011). Large-scale brain networks and psychopathology: a unifying triple network model. Trends in Cognitive Sciences 15, 483-506.

Mezulis AH, Abramson LY, Hyde JS, Hankin BL (2004). Is there a universal positivity bias in attributions? A meta-analytic review of individual, developmental, and cultural differences in the self-serving attributional bias. Psychological Bulletin 130, 711-747.

Minett TSC, Da Silva RV, Ortiz KZ, Bertolucci PHF (2008). Subjective memory complaints in an elderly sample: a cross-sectional study. International Journal of Geriatric Psychiatry 23, 49-54.

Mioshi E, Dawson K, Mitchell J, Arnold R, Hodges JR (2006). The Addenbrooke's Cognitive Examination Revised (ACE-R): a brief cognitive test battery for dementia screening. International Journal of Geriatric Psychiatry 21, 1078-1085.

Olssøn I, Mykletun A, Dahl AA (2005). The Hospital Anxiety and Depression Rating Scale: a cross-sectional study of psychometrics and case finding abilities in general practice. BMC Psychiatry 5, 46.

Pessoa L (2009). How do emotion and motivation direct executive control? Trends in Cognitive Sciences 13, 160-166.

Piccinelli M, Wilkinson G (2000). Gender differences in depression. British Journal of Psychiatry 177, 486-492.

Reid LM, MacLullich AM (2006). Subjective memory complaints and cognitive impairment in older people. Dementia and Geriatric Cognitive Disorders 22, 471-485.

Rock PL, Roiser JP, Riedel WJ, Blackwell AD (2014). Cognitive impairment in depression: a systematic review and meta-analysis. Psychological Medicine 44, 2029-2040.

Samplin E, Ikuta T, Malhotra AK, Szeszko PR, DeRosse P (2013). Sex differences in resilience to childhood maltreatment: effects of trauma history on hippocampal volume, general cognition and subclinical psychosis in healthy adults. Journal of Psychiatric Research 47, 1174-1179.

Schaakxs R, Comijs HC, Lamers F, Beekman ATF, Penninx B (2017). Age-related variability in the presentation of symptoms of major depressive disorder. Psychological Medicine 47, 543-552.

Schofield PW, Marder K, Dooneief G, Jacobs DM, Sano M, Stern Y (1997). Association of subjective memory complaints with subsequent cognitive decline in community-dwelling elderly individuals with baseline cognitive impairment. American Journal of Psychiatry 154, 609-615.

Shafto MA, Tyler LK, Dixon M, Taylor JR, Rowe JB, Cusack R, Calder AJ, Marslen-Wilson WD, Duncan J, Dalgleish T, Henson RN, Baryne C, Cam-CAN, Matthews FE (2014). The Cambridge Centre for Ageing and Neuroscience (Cam-CAN) study protocol: a cross-sectional, lifespan, multidisciplinary examination of healthy cognitive ageing. BMC Neurology 14, 204. 
Spalletta G, Piras F, Caltagirone C, Fagioli S (2014). Hippocampal multimodal structural changes and subclinical depression in healthy individuals. Journal of Affective Disorders 152, 105-112.

Sutin AR, Terracciano A, Milaneschi Y, An Y, Ferrucci L, Zonderman AB (2013). The trajectory of depressive symptoms across the adult life span. JAMA Psychiatry 70, 803-811.

Taquet M, Quoidbach J, de Montjoye Y-A, Desseilles M, Gross JJ (2016). Hedonism and the choice of everyday activities. Proceedings of the National Academy of Sciences 113, 9769-9773.

Taylor JR, Williams N, Cusack R, Auer T, Aguilar MA, Dixon M, Tyler LK, Cam-CAN, Henson RN (2017). The Cambridge Centre for Ageing and Neuroscience (Cam-CAN) data repository: structural and functional MRI, MEG, and cognitive data from a cross-sectional adult lifespan sample. NeuroImage 144, 262-269.
Watson LA, Dritschel B, Obonsawin MC, Jentzsch I (2007) Seeing yourself in a positive light: brain correlates of the self-positivity bias. Brain Research 1152, 106-110.

Wechsler D (1997). Manual for the Wechsler Adult Intelligence Scale-III (WAIS-III). The Psychological Corporation: San Antonio, TX.

Yates JA, Clare L, Woods RT (2017). Subjective memory complaints, mood and MCI: a follow-up study. Aging $\mathcal{E}$ Mental Health 21, 313-321.

Yates JA, Clare L, Woods RT, Matthews FE (2015). Subjective memory complaints are involved in the relationship between mood and mild cognitive impairment. Journal of Alzheimer's Disease 48, S115-S123.

Zigmond AS, Snaith RP (1983). The Hospital Anxiety and Depression Scale. Acta Psychiatrica Scandinavica 67, 361-370. 